\title{
Introduction to Sport, Emerging Media, and the Law
}

\author{
Galen Clavio \\ Indiana University
}

The landscape of emerging media is ever changing, with technological innovation and industry adaptation constantly pushing back the boundaries of the status quo. As sport industry leaders race to stay abreast, communication scholars and legal experts have often found it a challenge to keep pace. Sport media's core assumptions, which seemed inviolable 20 years ago, have been rocked by a series of unforeseen new communication channels, from social networks to mobile video, from the emerging spectator sport of video games to the rules and restrictions placed on world-class athletes' usage of social media for branding and marketing purposes.

As a sport media scholar who has spent the last decade researching emerging media in sport, I was particularly interested in reading the work of my fellow scholars and seeing their perspectives on the current wave of technological innovation. The landscape has changed considerably since I started my work in the mid-2000s, powered first by the emergence, and then the dominance, of handheld and Internet-enabled communication devices. This development naturally led to deeper establishments of digital identities for fans, athletes, and media members. Our current environment might have been predictable in 2006, but it would have been hard to describe in understandable terms back then. With a decade of emerging mobile media under our belts, my hope is that the articles in this special issue give scholars a sense of where things are headed in the next few years, and what factors and parameters need to be given heed in research and observation as we move forward.

I would like to express my sincere thanks to the entire editorial and organizational staff at the Journal of Legal Aspects of Sport, both for their willingness to devote a special issue to this important topic and for their unwavering support and assistance throughout the process. A special thanks goes out to journal editor Dr. Thomas A. Baker, who believed in the project from the beginning and worked with me to help make it a reality. I would also like to thank all of the reviewers who contributed their time and insight to the many manuscripts that were submitted for this issue. Your perspectives and expertise were invaluable in allowing the issue to feature such high-quality work.

This special issue focuses on emerging media topics that feature potential legal or regulatory gray areas. The articles in it are authored by an impressive array of

Clavio is with the National Sports Journalism Center at Indiana University, Bloomington. He can be reached at gclavio2@indiana.edu 
scholars, some coming with a background in traditional sport law and others with a background in sport and emerging media. I am pleased that this special issue presents a diverse set of perspectives, because I believe that the process of better understanding the legal or regulatory implications of emerging media in sport is best served by considering ideas from scholars of varied research interests.

The Aereo case held the promise of revolutionizing the sports broadcasting world for the mobile video age, and yet the traditional modes of game transmission managed to carry the day. Dittmore and Hutchens provide an overview of the case and the surrounding issues moving forward.

Twitter's social media star may have faded a bit in 2016, but the favored network for journalists, athletes, and opinion leaders continues to play an important role in sports media coverage and conversation. McKelvey and Grady focus their contribution to this special issue on the evolution of Twitter hashtag usage in the legal environment.

From Twitch.tv to the Big Ten Network to ESPN, e-sports made a sudden leap into the public consciousness of sports in 2016 and 2017. Holden, Kaburakis, and Rodenberg take us through a measured evaluation of the burgeoning world of e-sports and its potential policy and litigation implications.

Social media networks have brought many positive new aspects to fan-athlete interactions, but many negative aspects as well. Frederick's commentary on this subject examines some of the potential defamatory occurrences that have been directed at athletes in the recent past and evaluates some of the key areas to consider moving forward.

The Manti T'eo saga may be over four years old, but the market for social media impersonation and "catfishing" is still alive and worthy of further investigation. Smith, Smith, and Blaszka provide an examination of the processes used and the legal ramifications for athletes within the current social media environment.

The 2016 Summer Olympics in Rio de Janeiro saw social media playing its most prominent role ever in connecting fans across the globe to the games and the participating athletes. However, the IOC's rule structure surrounding marketing messages through social media created some interruptions in the flow of social media conversation. Schlereth and Frederick examine the current and future implications of these rules, and what they might mean moving forward for both the Olympics and for social media.

Taken together, these articles and commentaries provide fresh perspective on a variety of issues relating to emerging media, law, regulation, and policy, and serve as a launching pad for further scholarly investigation of these and related areas. My hope is that these articles find an audience among legal and sport communication scholars. I believe these two groups have much to offer each other in terms of understanding of their areas of specialty, and I think the combined efforts of both will contribute to a greater comprehension of emerging media in sport and its legal and regulatory parameters. 